\title{
Dynamics of a Pituitary Cell Model: Dependence on Long-Lasting External Stimulation and Potassium Conductance Kinetics
}

\author{
Takaaki Shirahata \\ Institute of Neuroscience, Kagawa School of Pharmaceutical Sciences, Tokushima Bunri University, Sanuki, \\ Japan \\ Email: tshi@kph.bunri-u.ac.jp
}

Received 10 April 2016; accepted 23 May 2016; published 26 May 2016

Copyright (C) 2016 by author and Scientific Research Publishing Inc.

This work is licensed under the Creative Commons Attribution International License (CC BY). http://creativecommons.org/licenses/by/4.0/

(c) (i) Open Access

\section{Abstract}

Stern et al. have developed a mathematical model describing pseudo-plateau bursting of pituitary cells. This model is formulated based on the Hodgkin-Huxley scheme and described by a system of nonlinear ordinary differential equations. In the present study, computer simulation analysis of this model was performed to evaluate the correlation between the dynamic states of the model and two system parameters: long-lasting external stimulation $\left(I_{a p p}\right)$ and the time constant of delayed-rectifier potassium conductance activation $\left(\tau_{n}\right)$. Computer simulation results revealed that the model showed four different dynamic states: a hyperpolarized steady state, a depolarized steady state, a repetitive spiking state, and a bursting state. An increase in $I_{a p p}$ changed the dynamic states from the hyperpolarized steady state to bursting state to depolarized steady state when $\tau_{n}$ was fixed at smaller values, whereas it changed the dynamic states from the hyperpolarized steady state to bursting state to repetitive spiking state when $\tau_{n}$ was fixed at larger values. An increase in $\tau_{n} 1$ ) did not change the dynamic states when $I_{a p p}$ was fixed at a very small value, 2) changed the dynamic states from the depolarized steady state to repetitive spiking state when $I_{a p p}$ was fixed at a very large value, and 3 ) changed the dynamic states from the depolarized steady state to bursting state to repetitive spiking state when $I_{a p p}$ was fixed at an intermediate value.

\section{Keywords}

Mathematical Model, Computer Simulation, Pituitary Cells, Pseudo-Plateau Bursting 


\section{Introduction}

Bursting is a dynamic state that appears in nonlinear dynamic systems such as neurons [1]. The bursting state is a condition in which a state of repetitive spikes and a state of quiescence alternately appear. The bursting dynamics of several neurons has been extensively studied via mathematical models, such as a model of bursting pacemaker neurons of the pre-Bötzinger complex [2] and the ghostbursting model of electrosensory neurons [3]. Both mathematical models are described by a system of nonlinear Ordinary Differential Equations (ODEs). In addition, bursting dynamics has been studied in a mathematical model (Stern model) that was developed by modification of a pituitary corticotroph cell model [4]. An important issue in the analysis of mathematical models that show bursting dynamics is clarification of the dependence of the dynamic states on a system parameter such as long-lasting external stimulation. For example, previous studies revealed that an increase in long-lasting external stimulation changes the dynamic states from quiescence to bursting to repetitive spiking in the preBötzinger complex neuron model [2], whereas it changes the dynamic states from quiescence to repetitive spiking to bursting in the ghostbursting model of electrosensory neurons [3]. However, how the dynamic states of the Stern model change in response to variations in external stimulation is not described in detail in a study by Stern et al. [4]. Although they have investigated the response of the model to transient external stimulation [4], they have not studied the response to long-lasting external stimulation.

Dynamic states of mathematical models that show bursting dynamics are affected by other system parameters, such as the time constant of potassium conductance. For example, changes in the time constant of dendritic delayed-rectifier potassium conductance inactivation induce a nonlinear effect on the dynamic states of the ghostbursting model [5]. In a mathematical model that has been derived based on pituitary lactotroph cell data, bursting dynamics appears when the time constant of fast-activating voltage- and calcium-dependent potassium (BK) conductance activation is fixed at a smaller value, whereas spiking dynamics appears when the time constant is fixed at a larger value [6]. In addition, it is implied that changes in the time constant of delayed-rectifier potassium conductance activation play an important role in modulating the dynamic states of the Stern model [4]. However, Stern et al. [4] have not revealed in detail how variations in the time constant values affect the dynamics of the model. Considering the previous studies described above, it is important to investigate the influence of variations in both long-lasting external stimulation and time constant on the dynamic states of the Stern model [4]. Therefore, the present study performed computer simulation analysis of the Stern model to reveal the dependence of the dynamic states on long-lasting external stimulation and time constant.

\section{Materials and Methods}

The Stern model analyzed in the present study is formulated based on the Hodgkin-Huxley formalism, and is described by a system of nonlinear ODEs. The model contains four state variables: the membrane potential of corticotroph cells $[V(t)(\mathrm{mV})][t$ is time $(\mathrm{s})]$, the activating variable of L-type calcium current $\left[m_{L}(t)\right]$, the activating variable of delayed-rectifier potassium current $[n(t)]$, and the concentration of calcium ions $[\mathrm{Ca}(t)(\mu \mathrm{M})]$. Time evolutions of these state variables are described:

$$
\begin{gathered}
C_{m} \frac{d V(t)}{d t}=I_{\text {app }}-I_{C a L}\left(V(t), m_{L}(t)\right)-I_{C a T}(V(t))-I_{K}(V(t), n(t))-I_{K C a}(V(t), C a(t))-I_{L}(V(t)) \\
\frac{d m_{L}(t)}{d t}=\frac{1}{\tau_{m L}(V(t))}\left(m_{L \infty}(V(t))-m_{L}(t)\right) \\
\frac{d n(t)}{d t}=\frac{1}{\tau_{n}}\left(n_{\infty}(V(t))-n(t)\right) \\
\frac{d C a(t)}{d t}=J_{\text {exchange }}(C a(t))+f b\left(J_{\text {influx }}\left(V(t), m_{L}(t)\right)-J_{\text {efflux }}(C a(t)),\right.
\end{gathered}
$$

in which $C_{m}$ is the membrane capacitance $(0.00314 \mathrm{nF}), I_{a p p}(\mathrm{pA})$ is the applied current corresponding to longlasting external stimulation (this parameter was varied from -1.8 to $2.0 \mathrm{pA}$ at an interval of $0.2 \mathrm{pA}$ ), $\tau_{n}(\mathrm{~s})$ is the time constant of activating variable of delayed-rectifier potassium current (this parameter was varied from 0.017 to $0.027 \mathrm{~s}$ at an interval of $0.001 \mathrm{~s}), f$ is the fraction of free calcium $(0.01), b$ is the ratio of cell surface area to 
cell volume $\left(0.6 \mu \mathrm{m}^{-1}\right)$, and $I_{C a L}\left(V(t), m_{L}(t)\right), I_{C a T}(V(t)), I_{K}(V(t), n(t)), I_{K C a}(V(t), C a(t)), I_{L}(V(t)), \tau_{m L}(V(t))$, $m_{L \infty}(V(t)), n_{\infty}(V(t)), J_{\text {exchange }}(C a(t)), J_{\text {influx }}\left(V(t), m_{L}(t)\right)$, and $J_{\text {efflux }}(C a(t))$ are the L-type calcium current, the T-type calcium current, the delayed-rectifier potassium current, the calcium-activated potassium current, the leak current, the time constant of activating variable of L-type calcium current, the steady state activation function of L-type calcium current, the steady state activation function of delayed-rectifier potassium current, the term for exchange between the cytosol and the internal store, the term for influx through calcium channels, and the term for pumping calcium out of the cell, respectively, each of which is calculated:

$$
\begin{gathered}
I_{C a L}\left(V(t), m_{L}(t)\right)=1.366\left(m_{L}(t)\right)^{2}(V(t)-60) \\
I_{C a T}(V(t))=0.001\left(\frac{1}{1+\mathrm{e}^{-(V(t)+45) / 8}}\right)^{2}\left(\frac{1}{1+\mathrm{e}^{(V(t)+52) / 5}}\right)(V(t)-60) \\
I_{K}(V(t), n(t))=4.1 n(t)(V(t)+80) \\
I_{\text {KCa }}(V(t), C a(t))=0.25\left(\frac{(C a(t))^{4}}{(C a(t))^{4}+0.5^{4}}\right)(V(t)+80) \\
I_{L}(V(t))=0.3(V(t)+50) \\
\tau_{m L}(V(t))=\frac{0.027}{\mathrm{e}^{(V(t)+60) / 22}+2 \mathrm{e}^{-2(V(t)+60) / 22}} \\
m_{L \infty}(V(t))=\frac{1}{1+\mathrm{e}^{-(V(t)+25) / 12}} \\
n_{\infty}(V(t))=\frac{1}{1+\mathrm{e}^{-(V(t)-5) / 8}} \\
J_{\text {exchange }}(C a(t))=\frac{0.1-C a(t)}{0.5} \\
J_{\text {efflux }}(C a(t))=40.0 \frac{(C a(t))^{2}}{(C a(t))^{2}+0.08^{2}} .
\end{gathered}
$$

Detailed explanations of the Stern model are provided elsewhere [4].

The open-source software Scilab (http://www.scilab.org/) was used to numerically solve ODEs. Initial conditions were $V(0)=-57.31515986286935 \mathrm{mV}, m_{L}(0)=0.06191856353928273, n(0)=0.0003852853926905176$, and $\mathrm{Ca}(0)=0.4861280925831973 \mu \mathrm{M}$.

\section{Results}

By systematically varying $I_{a p p}$ and $\tau_{n}$, we found that the Stern model had four different dynamic states: a hyperpolarized steady state, a bursting state, a depolarized steady state, and a repetitive spiking state. Examples of time courses of the membrane potential are shown in Figure 1 . Under conditions in which $\tau_{n}$ was $0.020 \mathrm{~s}$, the hyperpolarized steady state appeared in response to current injection of $I_{a p p}=-1.8 \mathrm{pA}$ (Figure 1(a)). When $I_{a p p}$ was $-1.0 \mathrm{pA}$, the dynamic state was the bursting state (Figure $1(\mathrm{~b})$ ). When $I_{\text {app }}$ was $1.8 \mathrm{pA}$, the dynamic state was the depolarized steady state (Figure $1(\mathrm{c})$ ). When $\tau_{n}$ was $0.027 \mathrm{~s}$ under conditions in which $I_{a p p}$ was $1.8 \mathrm{pA}$, the dynamic state was the repetitive spiking state (Figure 1(d)).

Figure 2 summarizes the dynamic states in a $\left(I_{a p p}, \tau_{n}\right)$-parameter space. We characterized the dynamic states of the Stern model by slicing the $\left(I_{a p p}, \tau_{n}\right)$-parameter space in a horizontal or a vertical direction. First, we sliced the parameter space in a horizontal direction between $\tau_{n}=0.022 \mathrm{~s}$ and $0.023 \mathrm{~s}$. When $\tau_{n}$ was $\leq 0.022 \mathrm{~s}$, an increase in $I_{\text {app }}$ with a value of $\tau_{n}$ being fixed changed the dynamic states from the hyperpolarized steady state to 


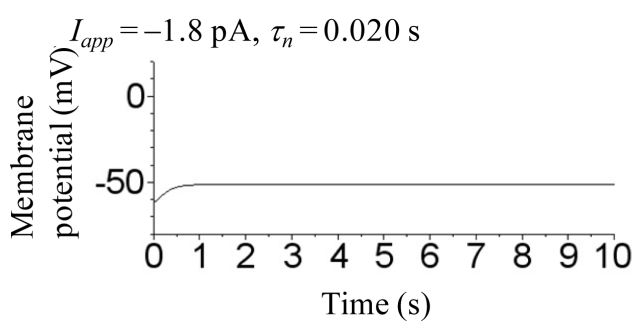

(a)

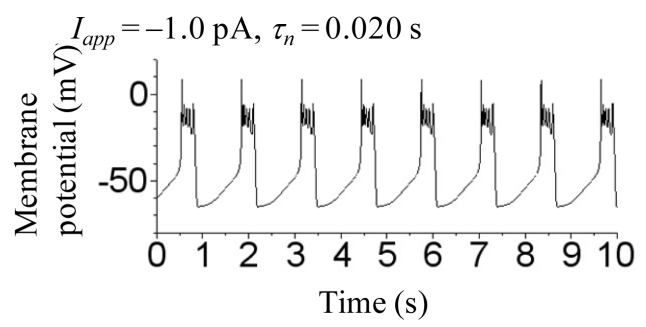

(b)

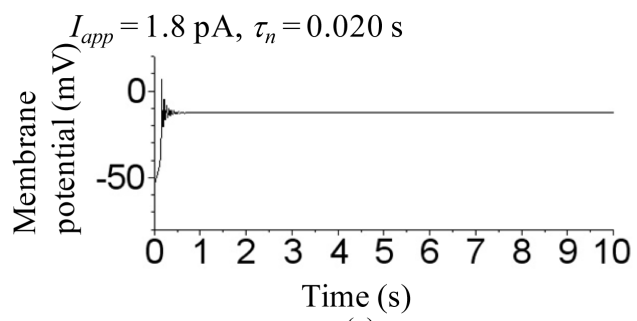

(c)

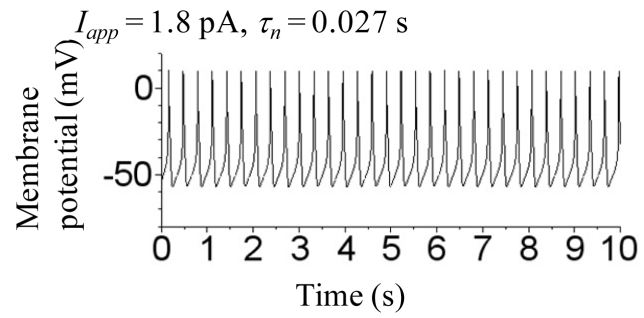

(d)

Figure 1. Time courses of the simulated membrane potentials of the Stern model. (a) The hyperpolarized steady state at ( $I_{a p p}$, $\left.\tau_{n}\right)=(-1.8 \mathrm{pA}, 0.020 \mathrm{~s})$; (b) The bursting state at $\left(I_{a p p}, \tau_{n}\right)=(-1.0 \mathrm{pA}, 0.020 \mathrm{~s})$; (c) The depolarized steady state at $\left(I_{a p p}, \tau_{n}\right)=$ $(1.8 \mathrm{pA}, 0.020 \mathrm{~s})$; (d) The repetitive spiking state at $\left(I_{a p p}, \tau_{n}\right)=(1.8 \mathrm{pA}, 0.027 \mathrm{~s})$. External stimulations $\left(i . e ., I_{a p p}\right)$ were continued from 0 to $10 \mathrm{~s}$.

bursting state to depolarized steady state. A decrease in $\tau_{n}$ from 0.022 did not change the $I_{a p p}$ threshold required for inducing the bursting state, whereas it decreased the $I_{a p p}$ threshold required for inducing the depolarized steady state. Therefore, a decrease in $\tau_{n}$ induced a decrease in the $I_{a p p}$ range in which the model showed the bursting state, finally resulting in a direct transition from the hyperpolarized steady state to depolarized steady state without undergoing the bursting state. When $\tau_{n}$ was $\geq 0.023 \mathrm{~s}$, an increase in $I_{a p p}$ with a value of $\tau_{n}$ being fixed changed the dynamic states from the hyperpolarized steady state to bursting state to repetitive spiking state. An increase in $\tau_{n}$ from 0.023 did not change the $I_{a p p}$ threshold required for inducing the bursting state, whereas it decreased the $I_{a p p}$ threshold required for inducing the repetitive spiking state. Therefore, an increase in $\tau_{n}$ induced a decrease in the $I_{a p p}$ range in which the model showed the bursting state, finally resulting in a direct transition from the hyperpolarized steady state to the repetitive spiking state without undergoing the bursting state.

Second, we sliced the parameter space in a vertical direction in two ways: 1 ) between $I_{a p p}=-1.8$ pA and $I_{a p p}=$ $-1.6 \mathrm{pA}$, and 2) between $I_{a p p}=1.8 \mathrm{pA}$ and $I_{a p p}=2.0 \mathrm{pA}$. Changes in the dynamic states can be characterized in three ways. First, when $I_{a p p}$ was $-1.8 \mathrm{pA}$, an increase in $\tau_{n}$ did not change the dynamic state: the state was the hyperpolarized steady state irrespective of the $\tau_{n}$ value. Second, when $I_{a p p}$ was $2.0 \mathrm{pA}$, an increase in $\tau_{n}$ changed 


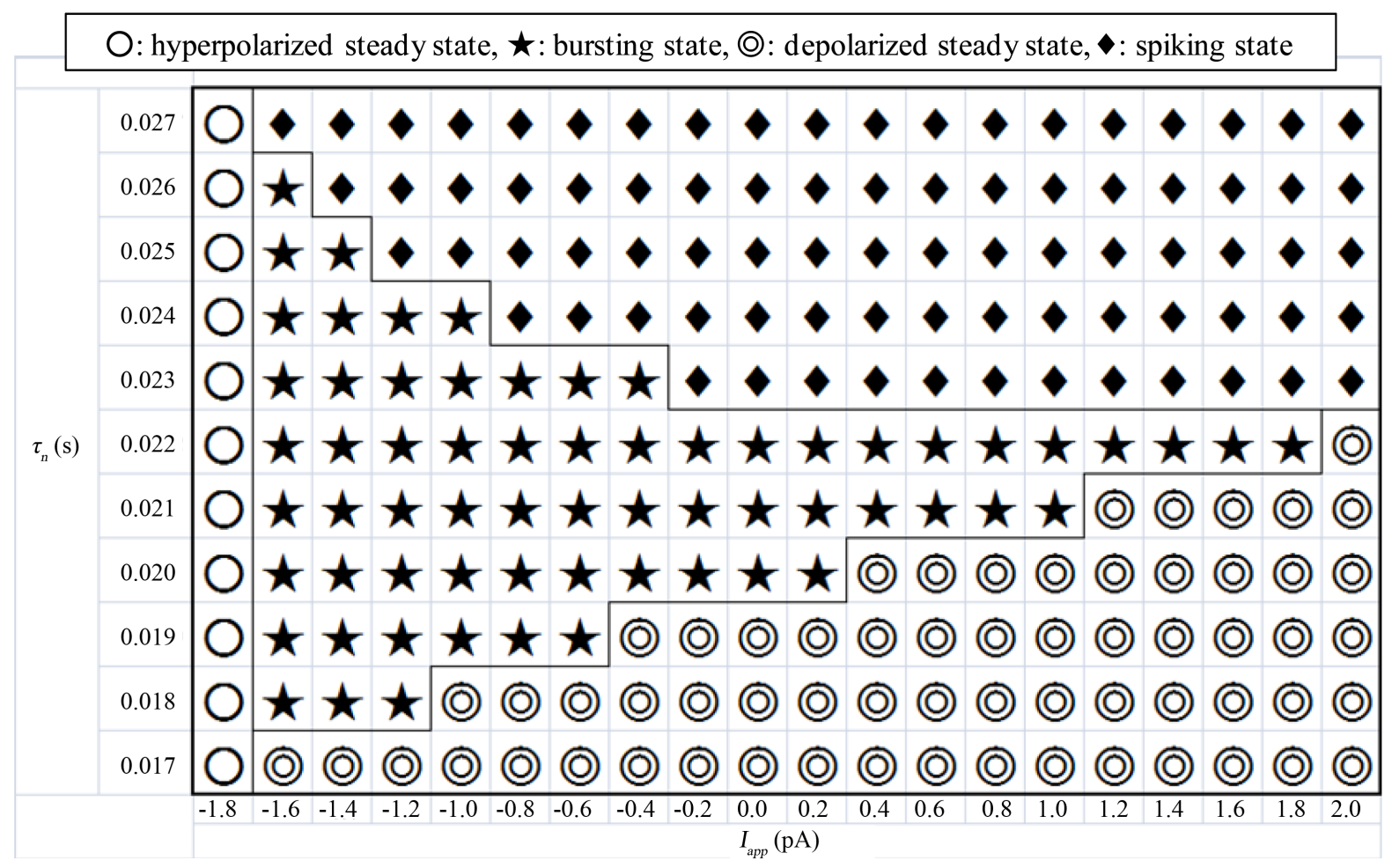

Figure 2. State diagram of the Stern model. The dynamic states of the model under various $\left(I_{a p p}, \tau_{n}\right)$ conditions are illustrated. ○: hyperpolarized steady state, $\star$ : bursting state, ○: depolarized steady state, $\diamond$ : repetitive spiking state.

the dynamic state from the depolarized steady state to repetitive spiking state. Third, when $I_{a p p}$ was between -1.6 and $1.8 \mathrm{pA}$, an increase in $\tau_{n}$ changed the dynamic state from the depolarized steady state to bursting state to repetitive spiking state. In addition, an increase in $I_{a p p}$ increased the $\tau_{n}$ threshold required for inducing the bursting state. In contrast, it decreased the $\tau_{n}$ threshold required for inducing the repetitive spiking state, and finally this threshold was constant as long as $I_{a p p}$ was a positive value. An increase in $I_{a p p}$ induced a decrease in the $\tau_{n}$ range in which the model showed the bursting state.

\section{Discussion}

In the present study, numerical simulation of the Stern model was performed [4], which revealed the dependence of the dynamic states on long-lasting external stimulation and the time constant of delayed-rectifier potassium conductance activation. Although Stern et al. have not only extensively performed analyses of the response of the model to transient external stimulation, but have also implied the importance of the time constant in modulating the dynamics of the model, they have neither performed analysis of the response of the model to long-lasting external stimulation nor described in detail the effect of variations in the time constant on the dynamics of the model [4]. An important contribution of the present study is that it can simultaneously resolve these two issues by varying two parameter values and visualizing the dynamic states in a two-dimensional parameter space. Although Stern et al. have described that an increase in the time constant changes the dynamic states from the bursting to repetitive spiking states, it is unclear whether this transition is affected by long-lasting external stimulation. The present study (Figure 2) reveals that the direction of the polarization of long-lasting external stimulation plays a different role in modulating the time constant threshold for the transition from the bursting to repetitive spiking states: the threshold under no stimulation can be smaller than that under longlasting external stimulation of a negative value (i.e., hyperpolarizing stimulation), whereas that under no stimulation is the same as that under long-lasting external stimulation of a positive value (i.e., depolarizing stimulation).

Previous studies have revealed that the time constant of potassium conductance plays an important role in the transition between the dynamic states in mathematical models of excitable cells. For example, variations in the 
time constant of dendritic delayed-rectifier potassium conductance inactivation induces the transition between the repetitive spiking and bursting states [5]. Variations in the time constant of BK conductance activation induce the transition between the repetitive spiking and bursting states [6]. Similarly to these two cases, the present study demonstrated that variations in the time constant were involved in the transition between the repetitive spiking and bursting states. However, the present study clarified the other two types of transition because of variations in the time constant: 1) the transition between the bursting and depolarized steady states and 2) the transition between the repetitive spiking and depolarized steady states.

\section{Conclusion}

The present study clarified four dynamic states of the Stern model [4] and the dependence of them on two system parameters (i.e., long-term external stimulation and time constant of delayed-rectifier potassium conductance activation) in detail. Furthermore, the hyperpolarizing, but not depolarizing, stimulation plays an important role in modulating the time constant threshold for the transition from the bursting to repetitive spiking states. In addition, variations in the time constant play an important role in various types of transition between the dynamic states.

\section{Acknowledgements}

The author would like to thank Enago (www.enago.jp) for the English language review.

\section{References}

[1] Izhikevich, E.M. (2006) Bursting. Scholarpedia, 1, 1300. http://dx.doi.org/10.4249/scholarpedia.1300

[2] Butera, R.J., Rinzel, J. and Smith, J.C. (1999) Models of Respiratory Rhythm Generation in the Pre-Bötzinger Complex. I. Bursting Pacemaker Neurons. Journal of Neurophysiology, 81, 382-397.

[3] Doiron, B., Laing, C., Longtin, A. and Maler, L. (2002) Ghostbursting: A Novel Neuronal Burst Mechanism. Journal of Computational Neuroscience, 12, 5-25. http://dx.doi.org/10.1023/A:1014921628797

[4] Stern, J.V., Osinga, H.M., LeBeau, A. and Sherman, A. (2008) Resetting Behavior in a Model of Bursting in Secretory Pituitary Cells: Distinguishing Plateaus from Pseudo-Plateaus. Bulletin of Mathematical Biology, 70, 68-88. http://dx.doi.org/10.1007/s11538-007-9241-x

[5] Shirahata, T. (2015) Evaluation of Kinetic Properties of Dendritic Potassium Current in Ghostbursting Model of Electrosensory Neurons. Applied Mathematics, 6, 128-135. http://dx.doi.org/10.4236/am.2015.61013

[6] Vo, T., Tabak, J., Bertram, R. and Wechselberger, M. (2014) A Geometric Understanding of How Fast Activating Potassium Channels Promote Bursting in Pituitary Cells. Journal of Computational Neuroscience, 36, 259-278. http://dx.doi.org/10.1007/s10827-013-0470-8 